\title{
EDITORIAL
}

\section{Paying Attention to Miss(ed) FITs}

\author{
Pascale M. White ${ }^{1}$. Steven H. Itzkowitz ${ }^{1}$
}

Accepted: 19 January 2021 / Published online: 20 February 2021

(C) The Author(s), under exclusive licence to Springer Science+Business Media, LLC part of Springer Nature 2021

The use of patient navigation (PN) improves colorectal cancer (CRC) screening participation. When it comes to screening colonoscopy completion, $\mathrm{PN}$ is particularly important due to the complexity of scheduling, preparing, and obtaining the procedure. For example, DeGroff reported in a randomized control trial that colonoscopy completion was significantly higher in the group that received navigation compared with patients who received usual care [1]. Effectiveness of PN is increased when using culturally tailored materials including language concordance with the target population [2]. PN can also increase compliance with screening colonoscopies among urban minorities, leading to the detection of clinically significant pathology [3]. Most studies using PN interventions have targeted patients who are screening naïve; few have studied patients who have already started their screening process with a noninvasive test, such as fecal immunochemical test (FIT). When offered a choice, many patients choose a noninvasive stool-based screening test in preference to colonoscopy. Yet, if such patients test positive and do not then undergo colonoscopy in a timely fashion, clinically important pathology is often overlooked. Though it has often been assumed that PN may not be needed for performing FIT testing, coordinating a timely diagnostic colonoscopy subsequent to a positive FIT can be as complex as getting a screening colonoscopy yet additionally imperative given the abnormal result.

In this issue of Digestive Diseases and Sciences, Cusumano et al. [4] report on the use of PN for follow-up of a positive FIT in a large urban healthcare system. The aim of the study was to investigate if $\mathrm{PN}$ increased the rate of diagnostic colonoscopy after a positive FIT and to report the barriers to follow-up despite navigation. A novelty of the study was the use of non-clinical patient navigators-individuals with college degrees who worked part-time in this

Steven H. Itzkowitz

steven.itzkowitz@mountsinai.org

1 The Dr. Henry D. Janowitz Division of Gastroenterology, Icahn School of Medicine at Mount Sinai, 1468 Madison Avenue, Box 1069, New York, NY 10029, USA program-who contacted patients via telephone to schedule the diagnostic colonoscopy. Of note, the time from the positive FIT test to the start of the PN process was 326 days. Their efforts resulted in $31 \%$ of FIT-positive patients completing their colonoscopy within 6 months. Though male gender and younger age were significant predictors of successful follow-up, overall $35 \%$ of patients declined diagnostic colonoscopy. Perhaps of even greater concern, in $35 \%$ of cases, the primary care provider declined to refer the patient for the diagnostic colonoscopy. The authors concluded that there were multilevel barriers to follow-up that persisted despite the implementation of PN.

Successful completion of any CRC screening test is fraught with patient and provider barriers. The patient barriers cited in this study included: concerns for increased procedural risk due to comorbidities, having a general hesitation about undergoing colonoscopy, low perceived priority or perceived lack of time to undergo the procedure, avoidance or fear of colonic preparation, inability to secure transportation, and perceived excessive costs. Although these barriers are similar to the barriers to colonoscopic CRC screening initiation [5], this study emphasizes that barriers to invasive testing are uncovered further along the screening continuum if left unaddressed. This highlights the need to reinforce to patients, even before they perform the FIT, that a colonoscopy is mandatory should the test be positive. The physician barriers underscored the gap between guideline recommendations and clinical practice. The most commonly cited reason was that many patients had a normal colonoscopy prior to the positive FIT. While this raises the question as to why FIT was even offered to those who had a recent colonoscopy, it is worth noting that the last colonoscopy occurred an average of 143-1686 days prior to the positive FIT test (mean 726 days). It also highlights that if a FIT is used as an interval exam between colonoscopies, physicians should realize that a positive FIT in this context warrants a repeat colonoscopy.

Similar gaffes in screening algorithms can be seen in other studies, which have also reported missed opportunities for referrals to diagnostic colonoscopy by physicians [6]. 
Indeed, this has prompted the United States Multi-Society Task Force to issue guidelines that specifically recommend a diagnostic colonoscopy after a positive FIT, outlining other quality metrics for FIT such as the adenoma detection rate (ADR) of colonoscopy for men and women [7]. Indeed, since more pathology is found when colonoscopies are performed due to a positive FIT, the expected ADRs should accordingly be higher. While a lack of knowledge of clinical guidelines are known physician barriers to CRC screening [8], a positive FIT should be a definitive turning point toward a diagnostic colonoscopy irrespective of a previously normal exam.

The timing of the diagnostic colonoscopy is important. Waiting longer than 6 months [9] and as much as $>9$ months [10] between a positive FIT test and diagnostic colonoscopy increases the likelihood of finding an advanced adenoma or malignancy. Of the $31 \%$ of patients who underwent the diagnostic colonoscopy in Cusumano's study, $2.7 \%$ had adenocarcinoma, $13.5 \%$ had advanced adenomas, $5.4 \%$ had sessile serrated polyp, and $27 \%$ had non-advanced adenomas. Although Selby et al. were successful in implementing system-level strategies for improving timely follow-up after a positive FIT [11], the strategies were implemented in patients 30 days after a positive FIT. Since Cusumano's patients had no coordinated follow-up testing for close to a year following the positive FIT, this population was harder to reach and the most at risk.

The study is impactful in that it gives insight into strategies for reaching a screening population that may be more refractory to diagnostic testing. Yet, if this population can complete a colonoscopy, one-third of the time with only patient-level outreach a year after their FIT, adding an additional layer of outreach at the systemic level may improve the completion rate.

As healthcare providers, due diligence is needed when we offer patients stool-based CRC screening. We should inquire as to the reason(s) that led them to choose this modality and address any barriers to invasive testing early on, in the event that it becomes necessary later. Initiating $\mathrm{PN}$ at the start of screening with FIT may make a difference, as providers could be more proactive in coordinating necessary resources if positive results are shown. Indeed, a challenging question is whether a patient should undergo a FIT if they are truly unlikely or unwilling to proceed with a diagnostic colonoscopy, since if a FIT test is done and a colonoscopy is not performed after a positive FIT, this constitutes a failed screening episode. It may be useful to explain to patients that by choosing FIT, they are signing up for a two-step screening process-a FIT followed by a colonoscopy if the FIT is positive, or a repeat FIT in one year if the FIT is negative. In the era of COVID-19, when many patients elect for at-home, noninvasive stool testing for CRC screening, it becomes even more essential to track all patients who test positive with FIT, confirm that the endoscopy units can accommodate the growing volume, and get them urgently navigated into timely colonoscopy. As gastroenterologists, we believe that the best CRC screening test is the one that gets done and gets done well. On a bright note, this study showed that even nonclinical patient navigators were effective a third of the time in getting hard-to-reach individuals in for colonoscopy. By the same token, this study is a reminder that more work is needed if the goal is to 'leave no positive FIT behind'.

\section{References}

1. DeGroff A, Schroy PC III, Morrissey KG et al. Patient navigation for colonoscopy completion: results of an RCT. Am J Prev Med. 2017;53:363-372

2. Jandorf L, Cooperman JL, Stossel LM et al. Implementation of culturally targeted patient navigation system for screening colonoscopy in a direct referral system. Health Educ Res. 2013;28:803-815

3. Jandorf L, Gutierrez Y, Lopez J, Christie J, Itzkowitz SH. Use of a patient navigator to increase colorectal cancer screening in an urban neighborhood health clinic. J Urban Health. 2005;82:216-224

4. Cusumano VT, Myint A, Corona E, et al. Patient navigation after positive fecal immunochemical test results increases diagnostic colonoscopy and highlights multilevel barriers to follow-up. Dig Dis Sci. (Epub ahead of print). https://doi.org/10.1007/s1062 0-020-6866-x.

5. Muthukrishnan M, Arnold LD, James AS. Patients' self-reported barriers to colon cancer screening in federally qualified health center settings. Prev Med Rep. 2019;15:100896

6. Singh H, Kadiyala H, Bhagwath $G$ et al. Using a multifaceted approach to improve the follow-up of positive fecal occult blood test results. Am J Gastroenterol. 2009;104:942-952

7. Robertson DJ, Lee JK, Boland CR et al. Recommendations on fecal immunochemical testing to screen for colorectal neoplasia: a consensus statement by the US Multi-society Task Force on colorectal cancer. Gastroenterology. 2017;152:1217-1237

8. White PM, Sahu M, Poles MA, Francois F. Colorectal cancer screening of high-risk populations: a national survey of physicians. BMC Res Notes. 2012;5:64

9. Lee YC, Fann JC, Chiang TH et al. Time to colonoscopy and risk of colorectal cancer in patients with positive results from fecal immunochemical tests. Clin Gastroenterol Hepatol. 2019;17:1332-1340

10. Forbes N, Hilsden RJ, Martel M et al. Association between time to colonoscopy after positive fecal testing and colorectal cancer outcomes: a systematic review. Clin Gastroenterol Hepatol. 2020;S1542-3565:31378-31381

11. Selby K, Jensen CD, Zhao WK et al. Strategies to improve followup after positive fecal immunochemical tests in a communitybased setting: a mixed-methods study. Clin Transl Gastroenterol. 2019;10:e00010

Publisher's Note Springer Nature remains neutral with regard to jurisdictional claims in published maps and institutional affiliations. 